\title{
Grain scale mechanisms for capillary collapse in a loose unsaturated pyroclastic soil
}

\author{
Sabatino Cuomo ${ }^{1, a}$, Mariagiovanna Moscariello ${ }^{1}$ and Simon Salager ${ }^{2}$ \\ ${ }^{1}$ University of Salerno, Department of Civil Engineering, Laboratory of Geotechnics, Italy \\ ${ }^{2}$ University Grenoble Alpes, INP Grenoble, 3SR Laboratory, France
}

\begin{abstract}
Soil collapse may occur passing from unsaturated to saturated conditions, thus causing major problems, among which one can mention a poor performance of the structures or the occurrence of landslides turning into flows. The mechanisms of the soil collapse have been studied at macroscopic scale since many years, while few observations at microscopic level are available. In this work, the mechanisms of capillary collapse were investigated for a volcanic (air-fall) pyroclastic soil of Southern Italy, which is characterized by an open metastable structure and is frequently involved into catastrophic rainfall-induced landslides. The experimental investigation was performed through X-ray Computed Tomography, which allows reconstructing 3D images of the specimen from the spatial distribution of the linear attenuation coefficient. The tests were carried out on coarse sand. During the tests, the specimens were loaded by its self-weight without any external load, and the suction was gradually reduced until the specimen collapse occurs. The aims of the experimental program were: i) follow the transformation of the specimen's microstructure; ii) evaluate the variation in terms of water content, porosity and grains spatial distribution; iii) analyse the effect of grain size distribution on the development of capillary forces and mass forces. The experimental evidences outline that, for the coarse pyroclastic sand, the collapse occurs at a very low suction, while it is not mandatory to reach the complete saturation.
\end{abstract}

\section{Introduction}

The collapse is commonly defined as the decrease in total volume of a soil resulting from the wetting-induced breakdown of its structure at essentially unchanging total vertical stress [1]. It occurs in granular soils, when capillary forces are of the same order of magnitude as other interparticle forces (i.e. skeleton forces, weight of particles, cementation) and when the microstructure is loose enough.

Several authors [2, 3, 4] studied the collapse that occurs in loose unsaturated soils and concluded that: i) the collapse deformation due to a suction reduction depends mainly upon the density and stress state under which it occurs; ii) the volume change due to a suction decrease mainly depends on the mean net stress and the initial void ratio, and the maximum collapse occurs when the mean net stress equals the initial yielding mean net stress of the unsaturated soil; iii) for unsaturated specimens compacted at the same initial density, the oedometric compression curves obtained for specimens saturated at different mean net stresses are more or less the same; the compression curve for initially unsaturated specimens wetted to zero suction is influenced by the value of initial density.

The interest for the collapse is that it causes various problems in geotechnical engineering, such as poor

\footnotetext{
a Corresponding author: scuomo@unisa.it
}

performance of the structures and the occurrence of rainfall-induced landslides. A significant example is represented by the landslides which involve the pyroclastic deposits of Southern Italy made of unsaturated soils originated from the explosive activity of the Somma-Vesuvius volcanic apparatus [5, 6, 7]. For these soils, an extensive data-set is available on the hydro-mechanical behaviour under both saturated and unsaturateted conditions. Indeed, the collapse of the pyroclastic soils was investigated at the specimen scale through wetting tests in triaxial and oedometric conditions. However, the capillary collapse and the modifications of the microstructure during the wetting process have been less observed. In this paper, the mechanism of capillary collapse was investigated for a pyroclastic soil of Southern Italy through the X-ray Computed Tomography, which is a non-destructive technique that allows visualizing the internal structure of a body through the measurement of density and atomic composition [8]. So far, the applications of the tomography to porous media have been mainly focused on: i) the analysis of porosity and fluid flow; ii) the evaluation of density, water content and volume fractions [9]; iii) the characterization of the microstructure of asphalts and concretes [8]; iv) the characterization of the local deformations within a soil specimen in a mechanical testing device [8]. 


\section{X-ray Computed Tomography}

The basic components of X-ray CT scanners are an X-ray source, a detector and a rotation system. Two possible configurations exist [10]: i) the X-ray source and the detector turn around the symmetry axis of the object (common for medical field); ii) the object turns around its symmetry axis while the detector is at a fixed place (common in the industrial field).

The experimental technique used in the present work was proposed by Arthur [11] and requires the acquisition of radiographs from different positions during a slow rotation of the specimen around its vertical symmetry axis.

After the acquisition, the images are processed - aimed to reconstruct the entire body volume - through the application of digital filters to improve the image quality and to reduce the artefacts related to the X-ray CT technique (i.e. misalignment of source, detector and axis of rotation; detector defects; anomalous bright pixels in the radiographs).

The reconstruction procedure provides several contiguous cross-sections, either vertical or horizontal, which allows $3 \mathrm{D}$ visualization of the selected features and the quantification of the $3 \mathrm{D}$ volumes

After the body reconstruction, the regions occupied by each phase can be recognized by visual interpretation obtaining qualitative estimates -, or using computational methods to obtain quantitative results [12]. The recognition of the phases which constitutes the object is commonly called "segmentation". Specifically, the segmentation consists into labelling each voxel (volumetric pixel) based on the ratio of its gray value to a reference threshold [13]. This is done referring to the "intensity histogram", which represents the number of voxels as a function of the intensity.

The segmentation is not an immediate process for geomaterials and in particular for unsaturated soils, which are composed by three main phases (grains, air and water). The histogram of an unsaturated soil is schematically composed of three normal distributions and therefore three peaks exist. Moreover, the tomographic images are obtained by absorption contrast, so it is possible to distinguish the phases by intensity or gray values. The low-density phases like air or water have lower intensity than solid grains; thus, the voxels occupied by air are darker than the solid voxels, while water has an intermediate gray level (Fig. 1).

The histograms of the different phases may overlap due to two artefacts: Gaussian Noise (GN) and Partial Volume Effect (PVE).

The GN is homogenously present in any image; while, the PVE is related to the existence of so called "mixels", i.e. voxels in which two or more phases are present. A mixel shows an intermediate grey value in between the two Gaussian curves, and the grey value depends on the percentage of each phase in the voxel. Consequently, a single threshold of the grey value is not sufficient to distinguish each phase properly.

To overcome the difficulties related to the CT images of unsaturated soils, a kind of region growing method is used [12]. The method was developed as one of region- based segmentation methods for digital images [14], and postulates that the neighboring voxel within one region have similar values [15]. The region-growing type segmentation is applied on 3D X-ray 8 bits (0-255 gray level range) reconstructed images. The procedure is composed of four steps: i) partial thresholding; ii) PVE filtering; iii) simultaneous phase growing; iv) filling of interface.

1) The "partial thresholding" consists in seeding the regions where each phase is present. The first step consists into selecting the regions, where the solid phase is certainly present. Then, the peaks of grains and air histograms are chosen. In this way, the grains threshold isolate the 50\% upper part of the grains Gaussian curve and the air threshold isolate the $50 \%$ lower part of the air Gaussian curve. For the middle Gaussian curve (water), the thresholds are defined to select a range centred at the peak of the water curve and extended $25 \%$ to the left (dark) and to the right (light) of the peak.

2) After the partial thresholding, four groups of voxels become visible: the dark phase (B1), the middle phase (B2), the bright phase (B3) and the remaining nonselected voxels (R), so it is possible to start the middle phase PVE filtering. The PVE consists of voxels that belongs to the group B2. A spherical selection around the voxels of B2 group can be used to distinguish the voxel of the middle phase and the PVE. The standard deviation of the voxels surrounding each voxel belonging to B2 group is used to separate the real middle-phase voxels from PVE voxels. The standard deviation of voxels representing PVE is higher than the voxel representing the middle phase, causing a small increase in the highest standard deviation values in the histogram. The voxels with high standard deviation (PVE voxels) are then excluded by using a threshold filtering of standard deviation. The threshold is taken at the inflexion point on the histogram. After PVE filtering, the voxels considered as PVE are excluded from B2 and put into R.

3) The simultaneous phase growing method consists of growing all three phases into the group of the remaining voxels $(\mathrm{R})$. At this stage, $\mathrm{R}$ represents the interface between the different phases and so they are not assigned to any phase. The growth stops when all the voxels belonging to $\mathrm{R}$ have at least two distinct phases as neighbours. When the phases are growing simultaneously, the first phase that "reaches" a new voxel incorporates it, but the phases do not "see" where they

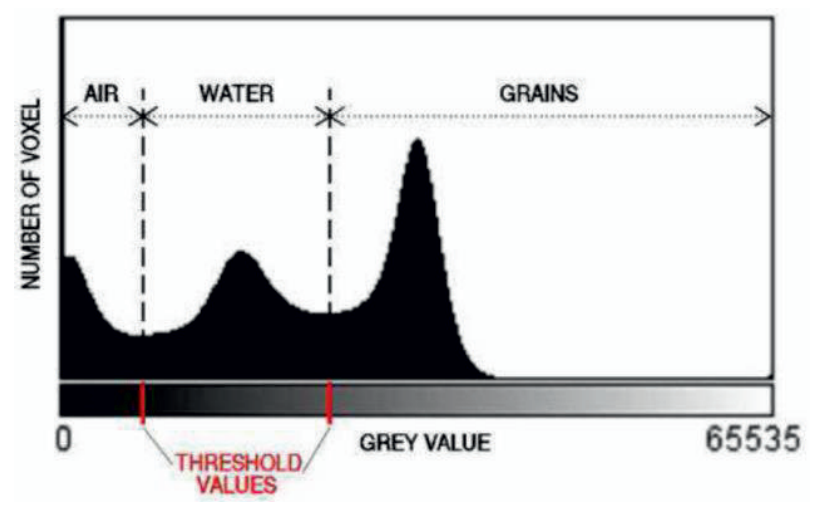

Figure 1. Histogram peaks for air, water and grains (Khaddour et al., 2013). 
grow. Hence, there is a potential for overgrowing in some areas of the image. These phenomena may be limited by choosing adequate partial threshold at the beginning of the procedure. Moreover, to avoid the growth of each phase in extreme gray values, a "tolerance threshold" is chosen for air, water and grain $\left(\mathrm{T}_{\text {air }}, \mathrm{T}_{1 \text {-water, }} \mathrm{T}_{2 \text {-water }}\right.$ and $\left.\mathrm{T}_{\text {grain }}\right)$. This means that the grey values where the phase is assumed as absent are excluded. These exclusions are determined by setting the gray value from which the Gaussian curve of each phase can be neglected. In this step, the PVE effect is also taken into account. After PVE filtering, some voxels (belonging to air or grain phases) of middle phase are not considered inside the partial threshold.

4) The final step of the procedure (interface filling) is to determine the phase to which the voxels of the interface are belonging. For each voxel at the interface, another sphere selects the surrounding voxels. The most present phase inside the sphere determines the phase segmentation. If two phases are in equal amount in the sphere, the considered voxels remain undecided. This procedure is repeated, and, usually after four times, the majority of the voxels have been filled. The remaining undecided voxels, instead, are defined with a simple threshold method and the phases are attributed as a function of the voxels grey level.

\section{Material and methods}

The sand used for the test was derived from a pyroclastic material of Southern Italy, which was formerly studied by [16], and classified as "class A ashy soil". The entire grain size distribution of the class A consists in 1.1 to $6.4 \%$ Gravel, 40.6 to $51.3 \%$ Sand, 40.9 to $53.61 \%$ Silt, 1.1 to $6.4 \%$ Clay. The grain shape is ranging from angular to sub-angular. The grain contains internal voids, so the soil specific gravity is quite small and equal to 2.55. The inter-particle porosity of undisturbed pyroclastic soil varies from 0.63 to 0.74 . These high values of porosity are related to the air-fall deposition, which conferred an "open" structure to the aggregates of soil particles [17].

For the specimen preparation, the pyroclastic soil was sieved and only the fraction with grain size included between $425 \mu \mathrm{m}$ and $600 \mu \mathrm{m}$ was kept. A mixture of sand and water was carried out at $20 \%$ of gravimetric water content, in order to obtain the loosest specimen. The mixture was put into a bag at least for two hours to ensure the correct hydration of the grains. The cylindrical specimen was prepared directly into the X-ray cabin. When the arrangement was built, the mixture was poured into the cell with a small spoon. During the preparation of the specimen, it was better not apply any type of load, in order to obtain a loose specimen, with the grains configuration more similar to undisturbed pyroclastic specimen. Then, the excess of soil was removed and the cell was covered. The cell was placed on the rotating table and connected to the suction controlled tank.

The tests were performed through the X-ray Tomograph of the 3SR Laboratoire at the Universite Joseph Fourier (Grenoble, France). The test apparatus was composed of: i) the cell; ii) the suction controller tank; iii) the rotating table; iv) the X-ray source; v) the X-ray detector (Fig.2). The need of high-resolution images imposed a relatively small size of both the specimen and cell. Indeed, the cell had a cylindrical shape $(10 \times 10 \mathrm{~mm}$, diameter and height $)$ and was built of acrylic fabric, which facilitates the penetration of X-rays. The apparatus was formerly designed and used to investigate the water retention behaviour of unsaturated materials (such as glass beads and Hostun sand) with X-ray tomography technique [18, 13]. The acquisition of radiographs and the control are carried out through the software RX-act developed by RX solutions (Annency, France).

Tab. 1 summarizes the X-ray scanning conditions. It is worth underlining that the voxel size was chosen near to technical upper limit of the X-ray source and detector, in order to acquire the images at high resolution. This was easily obtained approaching the cell and the X-ray source, but it implied that the other parameters of the test (like time of scanning, rotation rate and time of equalization) were selected in order to avoid disturbing the specimen.

During the test, the specimen was only subjected to its self-weight. The capillary pressure was controlled through the tank filled of deaired and demineralized water. The suction values were applied through the "negative water column" technique.

The first 3D image was acquired just after the specimen preparation to know the initial conditions and the internal structure of the specimen.

Then the suction was lowered from 0.5 to $0.1 \mathrm{kPa}$ in 4 steps $(0.5 \mathrm{kPa}, 0.3 \mathrm{kPa}, 0.2 \mathrm{kPa}$ and $0.1 \mathrm{kPa})$. The suction between two next steps was kept constant at least for 8 hours. This time lag was necessary for the specimen to reach the equalization and to remain stable during the acquisition. Once the equilibrium reached, the 3D X-ray images were acquired. The time necessary to acquire each 3D X-ray image was about 2 hours. For this test, the rotation of the specimen around its symmetry axis was slow in order to avoid any modification of specimen structure. The 3D images were reconstructed and then the segmentation of the images was carried out obtaining images composed of three gray values (trinarized images). Macroscopic porosity was calculated using the

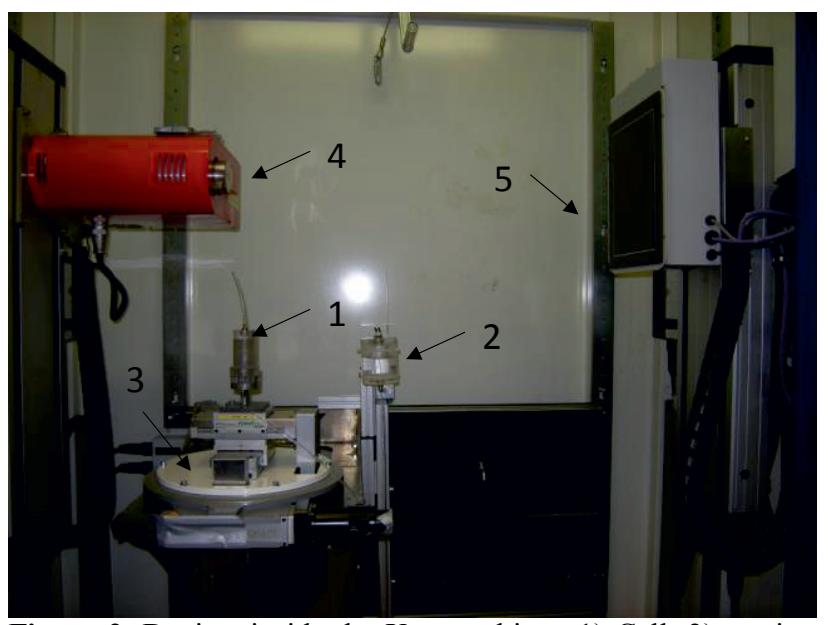

Figure 2. Device, inside the X-ray cabinet. 1) Cell; 2) suction controlled tank; 3) rotating table; 4) X-ray source; 5) detector. 
Table 1. Table of X-ray scanning conditions.

\begin{tabular}{ll}
\hline \# Projection & 1120 \\
Image Averaged & $5 / 10$ \\
Filter & Anti-ring shift \\
Voxel size & $7.56 \mu \mathrm{m}$ \\
\hline Source Voltage & $100 \mathrm{kV}$ \\
Source Current & $50 \mu \mathrm{A}$ \\
Time of scanning & $2 \mathrm{~h}$ \\
\hline
\end{tabular}

trinarized images. The 8-bits resulted trinarized images was of three gray values (64 for air, 128 for water, 192 for grain). The histograms of these images gave three values of the number of pixels, which were labelled for air $P_{\mathrm{a}}$, for water $P_{\mathrm{w}}$ and for grains $P_{\mathrm{g}}$. Through the number of pixels representing each phase, the porosity (n) was calculated through the Eq. 1.The numbers of pixels representing each phases was also used to estimate the degree of saturation $\left(S_{\mathrm{r}}\right)$ (Eq. 2) and the water content (Eq. 3).

$$
\begin{gathered}
n=\frac{\left(P_{g}+P_{w}+P_{a}\right)-P_{g}}{P_{g}+P_{w}+P_{a}} \\
S_{r}=\frac{P_{w}}{P_{w}+P_{a}} \\
\theta=S_{r} \cdot n
\end{gathered}
$$

\section{Results and discussion}

The capillary collapse phenomenon was investigated through a test on coarse pyroclastic sand. The specimen preparation procedure allowed reproducing the typical features of collapsible soils: an open structure, a high void ratio (or high porosity), low interparticle bond strength. Moreover, the angular shape of the grains allowed preserving the open, metastable structure. The initial configuration of the specimen was characterized by several aggregates of grains. The porosity was very high, because of the macro-voids that surrounded the aggregates. The Water Retention curve, in particular the main wetting curve, was evaluated using the same cell of figure 2 and the specimens were created through the above procedure and disassembled at each suction value investigated. The initial porosity was not equal at each steps of the main wetting curve and varied from 0.59 to 0.63 , this is a consequence of the specimen preparation procedure. The two curves (Fig. 3) did not overlap completely due to the different porosity of the specimens. The 3D X-ray images of the specimen at $0.5 \mathrm{kPa}$ and 0.3 $\mathrm{kPa}$ of suction show that the increment of water content did not influence the structure of the aggregates and the presence of macro-voids (Fig. 4). The specimen at 0.2 $\mathrm{kPa}$ of suction presented several transformations, the aggregates were not more separated by air, the water content increased and the macro-voids had different shape. The body forces produced only a little reduction of the height, but it was not uniform. The X-ray images at the suction of $0.1 \mathrm{kPa}$ show the collapse of the specimen.

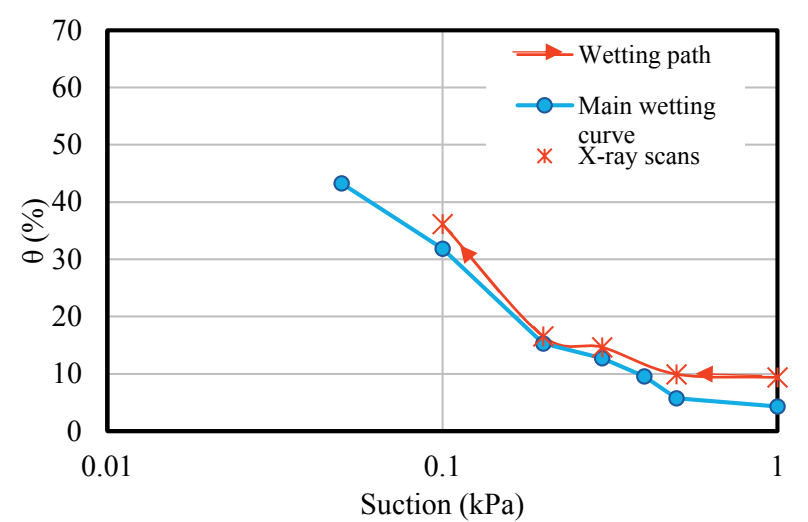

Figure 3. Wetting path during the test compared with the main wetting curve of the sand investigated.

In the correspondent image, the aggregates are not observable, but few air bubbles are still present even if the collapse happened. In figure 4 , the sequence of vertical slices at different section shows the evolution of the microstructure and the disappearance of the aggregates during the wetting. The reconstructed X-ray images of the test, consisting in 1500 horizontal slices, were trinarized using a Matlab code, which implemented the procedure illustrated in the Section 2.

The trinarized images presented some small black dots inside the grains. These dots represented some noise of the image. The procedure, used to clear the images, consisted of the despeackle of noise for each phase. Particularly, the grain and the water were separated in order to obtain images with only two phases: i) water and air; ii) grain and water. Later on, the two types of images were binarized and the despeackle tool was applied. Then, the two images were unified. The unified images clean of noise - were used to the quantitative analysis of the water content, degree of saturation and porosity. The
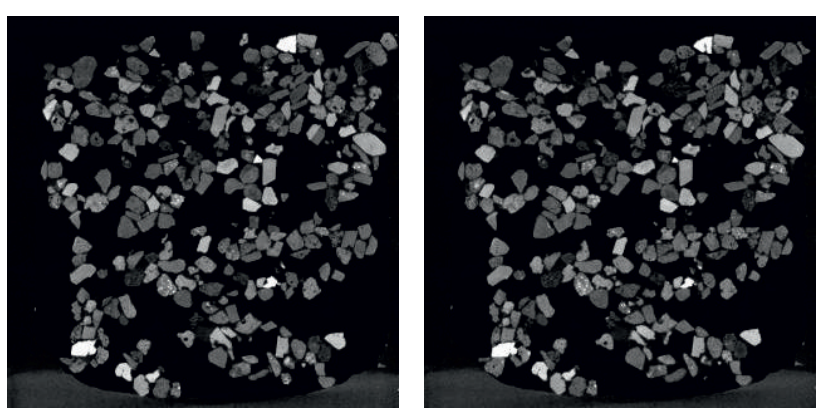

Initial conditions

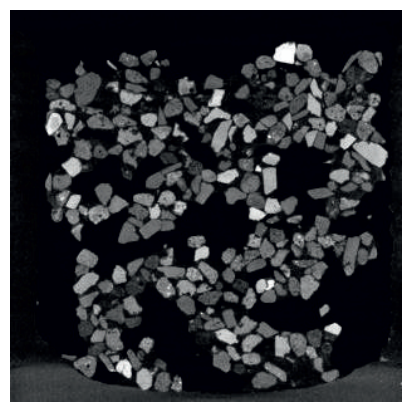

Suction $=0.2 \mathrm{kPa}$
Suction $=0.3 \mathrm{kPa}$

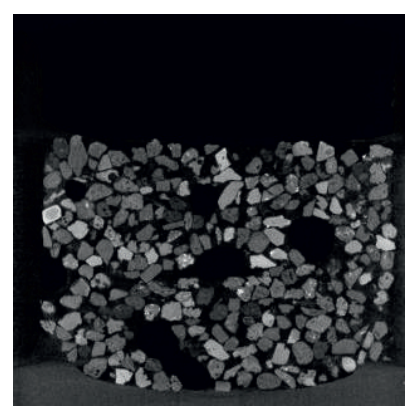

Suction $=0.1 \mathrm{kPa}$

Figure 4. X-ray $\mathrm{CT}$ images of the vertical section $\mathrm{N}^{\circ} 750$ during the wetting test. 


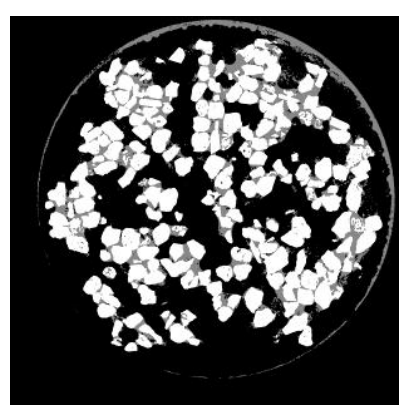

Initial conditions

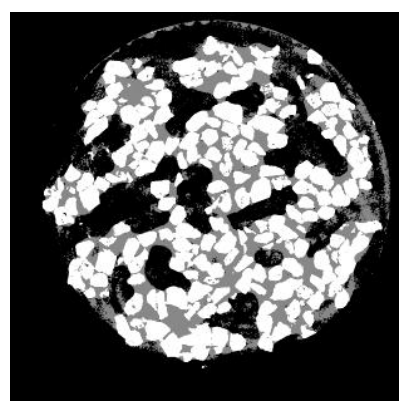

Suction $=0.2 \mathrm{kPa}$

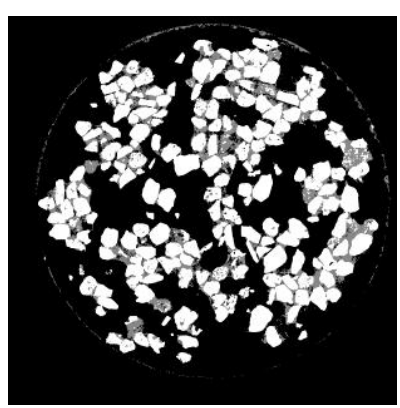

Suction $=0.3 \mathrm{kPa}$

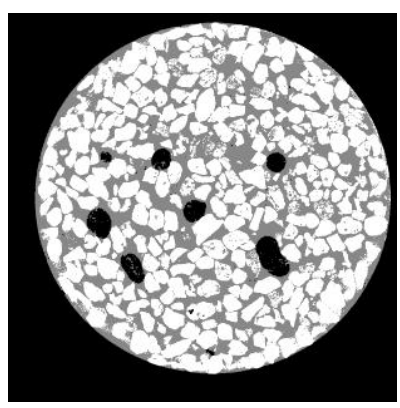

Suction $=0.1 \mathrm{kPa}$

Figure 5. Trinarized images for Horizontal slice $\mathrm{N}^{\circ} 750$, at the middle of the specimen.

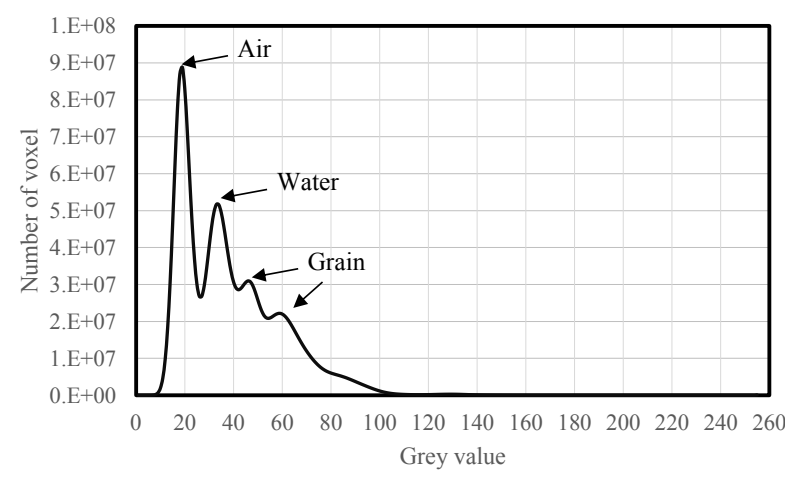

Figure 6. Histogram corresponding to the scan carried out at the collapse. The three phases and the correspondent peaks are clearly observable.
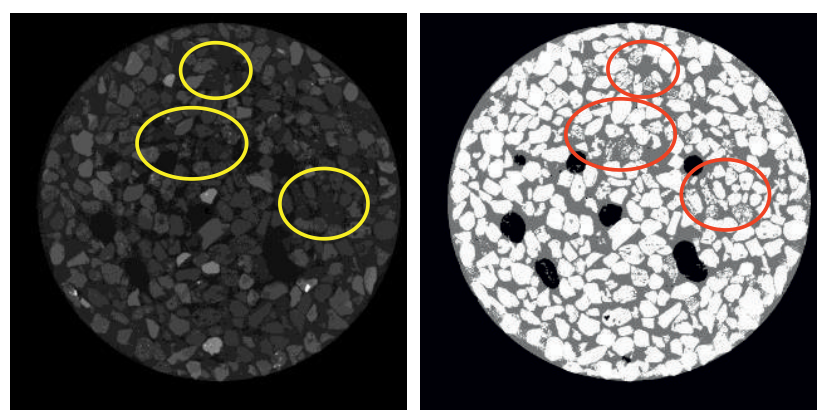

Figure 7. Comparison between the original image (left) and the trinarized image (right) of the scan carried out at $0.1 \mathrm{kPa}$ of suction (scan corresponds to the collapse of the specimen).

pyroclastic sand, because of its volcanic origin, is made of grains not homogeneous, which have different "adsorption capacity" (density), so that the histogram is quite different from the typical histogram of a three phases material (Fig.1). The histogram concerning the scans at suction $0.1 \mathrm{kPa}$ is plotted in figure 6 to show the

Table 2. Porosity $(n)$, water content $(\theta)$ and saturation degree $\left(S_{\mathrm{r}}\right)$ evaluated from the X-ray images of the horizontal slice $\mathrm{N}^{\circ} 750$ of figure 4 , compared to standard measurements.

\begin{tabular}{cccccc}
\hline$\#$ & $\begin{array}{c}\text { Suction } \\
(\mathrm{kPa})\end{array}$ & $\begin{array}{c}n_{s t} \\
(\%)\end{array}$ & $\begin{array}{c}n \\
(\%)\end{array}$ & $\begin{array}{c}\theta \\
(-)\end{array}$ & $\begin{array}{c}S_{r} \\
(\%)\end{array}$ \\
\hline 1 & $\mathrm{I} . \mathrm{C}$ & 72 & 67 & 0.08 & 10 \\
2 & 0.5 & 72 & 66 & 0.10 & 15 \\
3 & 0.3 & 71 & 65 & 0.12 & 19 \\
4 & 0.2 & 69 & 59 & 0.24 & 42 \\
5 & 0.1 & 54 & 40 & $0.35^{*}$ & $88^{*}$ \\
$*$ Measurements through & standard & procedure: & $\theta=0.39$, \\
$S_{r}=0.71$, and $n_{s t}$ column.
\end{tabular}

main differences towards a common three phases specimen. The histogram of figure 6 shows one peak for air, one for water and two peaks for the solid phase due to the different density of the grains.

The grains with a grey level close to that of the water correspond to little pumices with a high internal porosity and low specific weight. The grains with high grey value instead have a low value of internal porosity.

The presence of grains with gray level close to water phase influenced the results of the trinarization and so the estimate of the main physical properties (i.e. water content, saturation degree, porosity). For example, figure 7 shows the comparison between the original image and the result of the trinarization. The two images correspond to the horizontal section in the middle of the specimen at collapse. Yellow and red circles (Fig.7) correspond to the grains, which are entirely visible in the original image, while are partially or totally computed as water in the trinarized image. The soil porosity evaluated through the trinarization of the images of figure $5\left(n_{r x}\right)$ - for the horizontal cross-section at the middle height specimen was compared to the soil porosity calculated through the standard measurement of the specimen height during the tests $\left(n_{s t}\right)$, and they are both reported in Table 2 .

The differences are attributable to: i) the presence of "mixels", which may affect the evaluation of the solid phase amount; ii) to the different procedure to evaluate the height of the specimen; iii) and, more importantly, to the inhomogeneties induced in the specimen by the wetting process.

\section{Conclusions}

The capillary collapse of coarse volcanic sand was investigated using the X-ray Computed Tomography. This non-destructive full-field method allowed following the internal changes of the specimen during a wetting test. The images recorded were used to obtain the degree of attenuation of the X-rays, which is related to the material composition and energy of the source. These images, after the reconstruction and segmentation procedures, furnished qualitative and quantitative information about internal structure, porosity, water content, saturation degree, but also about intergranular forces, stresses and displacements.

The qualitative description of the changes in the internal structure of the specimen and the variation of main physical properties (porosity, water content and 
saturation degree) during the wetting test were discussed. Particularly, the specimen showed a double porosity (void among the grains and void among the aggregates), which collapsed only during the saturation of the biggest macro-pores when capillary bridges between grain clusters merged. One peculiarity of the soil behaviour was the presence of air bubbles at collapse. This was confirmed by the saturation degree, which did not reach unity during the collapse. The test underlined that the capillary bridges were enough strong so that the aggregates preserved their structure until suction reaches $0.2 \mathrm{kPa}$, corresponding to a $30 \%$ increment of the saturation degree, from 10 to $42 \%$. In conclusion, quantitative measurements were confidently achieved for water content and porosity, and in addition the experimental evidences outline that for the coarse pyroclastic sand the collapse occurs at a very low suction, while it is not mandatory to reach the complete saturation.

\section{Acknowledgements}

This research activity was developed within the framework of 2014-2015 Erasmus+ European Project and 2015-2016 Galileo Project Campus France "Soil mechanical behaviour from grain to specimen scale laboratory testing: towards new sustainable mitigation works against flow-like landslides and similar phenomena related to climate change" Grant n. G15-110.

\section{References}

1. Lawton, E. C., Fragaszy, R. J., Hardcastle, J. H., Stress ratio effects on collapse of Compacted Clayey sand. Journal of Geotechnical Engineering, ASCE, 117(5), 714-730 (1991).

2. Jennings, J.E.B., Burland, J.B., Limitations to the use of effective stresses in partly saturated soils. Géotechnique, 12(2), 125-144 (1962).

3. Kato, S., Kawai, K., Deformation characteristics of a compacted clay in collapse under isotropic and triaxial stress state. Soils Foundation, 40(5), 75-90 (2000).

4. Sun, D., Sheng, D., Xu,Y., Collapse behaviour of unsaturated compacted soil with different initial densities. Canadian Geotechnical Journal, 44(6), 673-686 (2007).

5. Cascini, L., Cuomo, S., Guida, D., Typical source areas of May 1998 flow-like mass movements in the Campania region, Southern Italy. Engineering Geology 96(3), 107-125 (2008).

6. Cascini, L., Cuomo, S., Pastor, M., Sorbino, G., Modelling of rainfall-induced shallow landslides of the flow-type. ASCE's Journal of Geotechnical and Geoenvironmental Engineering, 1, 85-98 (2010).

7. Cascini, L., Cuomo, S., Pastor, M., Sacco, C., Modelling the post-failure stage of rainfall-induced landslides of the flow-type, Canadian Geotechnical
Journal , 50(9), 924-934 (2013).

8. Viggiani, G., Hall, S. A., Full-field measurements in experimental geomechanics: historical perspective, current trends and recent results. ALERT Doctoral School 2012 Advanced experimental techniques in geomechanics (2012). Available at http://www.alertgeomaterials.eu/internal/2012/school /2012_ALERT_school.pdf

9. Riede-, I., Characterization and micro-scale analysis of Hostun sand water retention behaviour. MSc, Université de Grenoble, 216 pages (2011).

10. Baruchel, J., Buffière, J-Y., Maire, E., Merle, P., Peix, G., X-ray Tomography in Material Science. Hermes Science Publications, Paris, (2000).

11. Arthur, J.R.F., New techniques to measure new parameters. Proceedings of Roscoe Memorial Symposium o Stress-Strain Behaviour of Soils, Cambridge, G.T. Foulis \& Co., 340-346 (1971).

12. Hashemi, M. A., Khaddour, G., Francois, B., Massart, T. J., Salager, S., A tomographic imagery segmentation methodology for three-phase geomaterials based on simultaneous region growing. Acta Geotechnica, 9, 831-846 (2014).

13. Khaddour, G., Hashemi, A., Salager, S., Desrues, J., Massart, J., Francois, B., Phase segmentation in 3D $\mathrm{X}$-ray images of unsaturated granular materials. Proceedings of XVI French-Polish Colloquium of Soil and Rock Mechanics, (2013).

14. Rosenfeld, A., Kak, A. C., Digital Picture Processing, Academic Press, Inc. Orlando, FL, USA (1982).

15. Adams, R., Bischof, L., Seeded region growing. Pattern Analysis and Machine Intelligence, IEEE Transactions 16(6), 641-647 (1994).

16. Bilotta, E., Cascini, L., Foresta, V., and Sorbino, G.. Geotechnical characterization of pyroclastic soils involved in huge flowslides. Geotechnical and Geological Engineering, 23, 365-402 (2005).

17. Lancellotta, R., Di Prisco, C., Costanzo, D., Foti, S., Sorbino, G., Buscarnera, G., Cosentini, R.M., Foresta, V. Caratterizzazione e modellazione geotecnica. In: Criteri di zonazione della suscettibilità e della pericolosità da frane innescate da eventi estremi (piogge e sisma). Leonardo Cascini. Composervice srl, Padova, 266-319 (in Italian). ISBN 9788890687334 (in Italian) (2012).

18. Riedel, I., Andò, E., Salager, S., Bésuelle, P and Viggiani, G., Water retention behaviour explored by X-ray CT analysis, Unsaturated Soils: Research and Applications, 1, Proceedings of the 2nd European Conference on Unsaturated Soils, 20-22 June 2012, Napoli, Italy, Springer, 81-88 (2012). 